\title{
SOBOLEV SPACES, FINE GRADIENTS AND QUASICONTINUITY ON QUASIOPEN SETS
}

\author{
Anders Björn, Jana Björn and Visa Latvala \\ Linköping University, Department of Mathematics \\ SE-581 83 Linköping, Sweden; anders.bjorn@liu.se \\ Linköping University, Department of Mathematics \\ SE-581 83 Linköping, Sweden; jana.bjorn@liu.se \\ University of Eastern Finland, Department of Physics and Mathematics \\ P. O. Box 111, FI-80101 Joensuu, Finland; visa.latvala@uef.fi
}

\begin{abstract}
We study different definitions of Sobolev spaces on quasiopen sets in a complete metric space $X$ equipped with a doubling measure supporting a $p$-Poincaré inequality with $1<$ $p<\infty$, and connect them to the Sobolev theory in $\mathbf{R}^{n}$. In particular, we show that for quasiopen subsets of $\mathbf{R}^{n}$ the Newtonian functions, which are naturally defined in any metric space, coincide with the quasicontinuous representatives of the Sobolev functions studied by Kilpeläinen and Malý in 1992 .
\end{abstract}

\section{Introduction}

We study different definitions of Sobolev functions on nonopen sets in metric spaces. Even in $\mathbf{R}^{n}$, it is not obvious how to define Sobolev spaces on nonopen subsets, but fruitful definitions have been given on quasiopen sets $U$, i.e. on sets which differ from open sets by sets of arbitrarily small capacity. Kilpeläinen-Malý [12] gave the first definition of $W^{1, p}(U)$ in 1992 by means of quasicovering patches of global Sobolev functions. More recently, Sobolev spaces, and in particular so-called Newtonian spaces, have been studied on metric spaces. Thus by considering $U$ as a metric space in its own right (and forgetting the ambient space) we get another candidate, the Newtonian space $N^{1, p}(U)$.

The main purpose of this paper is to show that for quasiopen sets $U \subset \mathbf{R}^{n}$, the two spaces $W^{1, p}(U)$ and $N^{1, p}(U)$ coincide, with equal norms. To be precise, the functions in $N^{1, p}(U)$ are more exactly defined than a.e., and we have the following result.

Theorem 1.1. Let $U \subset \mathbf{R}^{n}$ be quasiopen, and let $u: U \rightarrow[-\infty, \infty]$ be an everywhere defined function. Then $u \in N^{1, p}(U)$ if and only if $u \in W^{1, p}(U)$ and $u$ is quasicontinuous. Moreover, in this case $\|u\|_{N^{1, p}(U)}=\|u\|_{W^{1, p}(U)}$.

On open sets in $\mathbf{R}^{n}$, the equality between the Newtonian and Sobolev spaces was proved by Shanmugalingam [16]. The proof of Theorem 1.1 is quite involved, and we will need most of the results in this paper to deduce it. We will also use several results related to the fine topology from our earlier papers [2]-[4].

The metric space approach to Sobolev functions makes it in principle possible to consider Sobolev spaces and variational problems on arbitrary sets, but it turns

doi:10.5186/aasfm.2016.4130

2010 Mathematics Subject Classification: Primary 46E35; Secondary 30L99, 31C40, 31C45, $31 \mathrm{E} 05$.

Key words: Fine gradient, fine topology, metric space, minimal upper gradient, Newtonian space, quasicontinuous, quasiopen, Sobolev space. 
out that there is not much point in considering more general sets than the quasiopen ones. More precisely, in [2] it was shown that the Dirichlet problem for $p$-harmonic functions in an arbitrary set coincides with the one in the set's fine interior. Moreover, the spaces of Sobolev test functions with zero boundary values are the same for the set and its fine interior. Finely open sets are quasiopen and quasiopen sets differ from finely open sets only by sets of capacity zero [4]. The fine topology, consisting of the finely open sets, is the coarsest topology making all superharmonic functions continuous [3] and serves as a tool for many deep properties in potential theory.

On the other hand, it is natural to study Sobolev spaces on quasiopen sets, as these sets have enough structure to carry reasonable families of Sobolev functions, and in particular of test functions. This is important for studying partial differential equations and variational problems on such sets, see Kilpeläinen-Malý [12] and Latvala [14]. The results in [2] also show that restrictions of (upper) gradients from the underlying space ( $\operatorname{such}$ as $\mathbf{R}^{n}$ ) behave well on quasiopen sets, but not on more general sets.

Quasiopen sets can be very different from the usual open sets, the simplest examples are open sets with an arbitrary set of zero capacity removed or added. Such a removed set can be dense, causing the interior of the resulting set to be empty. From the point of view of potential theory, such a set behaves like the original open one. A typical finely open set is the complement of the Lebesgue spine in $\mathbf{R}^{3}$ with the tip of the spine added to it. This is natural, since for harmonic and superharmonic functions, the tip behaves more like an interior point than a boundary point. More generally, finely open sets contain points which have only a "thin" connection with the complement of the set. See also Examples 9.5 and 9.6 in [2].

As hinted before Theorem 1.1, Newtonian functions are better representatives than the usual Sobolev functions. Namely, it was shown by Björn-Björn-Shanmugalingam [6], that all Newtonian functions on open sets are quasicontinuous. Moreover, they are finely continuous outside of sets of zero capacity, by J. Björn [7] or (independently) Korte [13]. We extend both these results to quasiopen sets. For these results, as well as for Theorem 1.2, it is assumed that the assumptions stated at the beginning of Section 3 are satisfied.

Theorem 1.2. Let $U$ be quasiopen, and $u \in N^{1, p}(U)$. Then $u$ is quasicontinuous in $U$ and finely continuous quasieverywhere in $U$.

This will be used as an important tool when establishing Theorem 1.1.

Acknowledgement. The first two authors were supported by the Swedish Research Council. Part of this research was done during several visits of the third author to Linköping University in 2012-2015 and while all three authors visited Institut MittagLeffler in the autumn of 2013. We thank both institutions for their hospitality and support.

\section{Notation and preliminaries}

In this section we will introduce and give precise definitions of the necessary metric space concepts used in this paper. We will be very brief, and refer to BjörnBjörn-Latvala [3] and [4] for more extensive introductions. See also the monographs Björn-Björn [1] and Heinonen-Koskela-Shanmugalingam-Tyson [10], where the theory is thoroughly developed with proofs.

We assume throughout the paper that $X=(X, d, \mu)$ is a metric space equipped with a metric $d$ and a positive complete Borel measure $\mu$ such that $0<\mu(B)<\infty$ for 
all (open) balls $B \subset X$. We also assume that $1<p<\infty$. The measure $\mu$ is doubling if there exists $C>0$ such that for all balls $B=B\left(x_{0}, r\right):=\left\{x \in X: d\left(x, x_{0}\right)<r\right\}$ in $X$, we have $0<\mu(2 B) \leq C \mu(B)<\infty$, where $\lambda B=B\left(x_{0}, \lambda r\right)$. A curve is a continuous mapping from an interval, and a rectifiable curve is a curve with finite length. We will only consider curves which are nonconstant, compact and rectifiable. A curve can thus be parameterized by its arc length $d s$.

Definition 2.1. A measurable function $g: X \rightarrow[0, \infty]$ is a $p$-weak upper gradient of $f: X \rightarrow[-\infty, \infty]$ if for $p$-almost every curve $\gamma:\left[0, l_{\gamma}\right] \rightarrow X$,

$$
\left|f(\gamma(0))-f\left(\gamma\left(l_{\gamma}\right)\right)\right| \leq \int_{\gamma} g d s
$$

where the left-hand side is $\infty$ if at least one of the terms therein is infinite. A property holds for $p$-almost every curve if the curve family $\Gamma$ for which it fails has zero $p$-modulus, i.e. there is $\rho \in L^{p}(X)$ such that $\int_{\gamma} \rho d s=\infty$ for every $\gamma \in \Gamma$.

If $f$ has a $p$-weak upper gradient in $L_{\text {loc }}^{p}(X)$, then it has a minimal $p$-weak upper gradient $g_{f} \in L_{\mathrm{loc}}^{p}(X)$ in the sense that for every $p$-weak upper gradient $g \in L_{\mathrm{loc}}^{p}(X)$ of $f$ we have $g_{f} \leq g$ a.e.

Definition 2.2. Let for measurable $f$,

$$
\|f\|_{N^{1, p}(X)}=\left(\int_{X}|f|^{p} d \mu+\inf _{g} \int_{X} g^{p} d \mu\right)^{1 / p}
$$

where the infimum is taken over all $p$-weak upper gradients $g$ of $f$. The Newtonian space on $X$ is

$$
N^{1, p}(X)=\left\{f:\|f\|_{N^{1, p}(X)}<\infty\right\} .
$$

The space $N^{1, p}(X) / \sim$, where $f \sim h$ if and only if $\|f-h\|_{N^{1, p}(X)}=0$, is a Banach space and a lattice. In this paper we assume that functions in $N^{1, p}(X)$ are defined everywhere (with values in $[-\infty, \infty]$ ), not just up to an equivalence class in the corresponding function space.

For a measurable set $E \subset X$, the Newtonian space $N^{1, p}(E)$ is defined by considering $\left(E,\left.d\right|_{E},\left.\mu\right|_{E}\right)$ as a metric space in its own right. For an arbitrary set $A \subset X$, we let

$$
N_{0}^{1, p}(A)=\left\{\left.f\right|_{A}: f \in N^{1, p}(X) \text { and } f=0 \text { on } X \backslash A\right\} .
$$

Functions from $N_{0}^{1, p}(A)$ can be extended by zero in $X \backslash A$ and we will regard them in that sense if needed.

The capacity of an arbitrary set $E \subset X$ is

$$
C_{p}(E)=\inf _{u}\|u\|_{N^{1, p}(X)}^{p},
$$

where the infimum is taken over all $u \in N^{1, p}(X)$ such that $u \geq 1$ on $E$. A property holds quasieverywhere (q.e.) if the set of points for which it fails has capacity zero. The capacity is the correct gauge for distinguishing between two Newtonian functions. If $u \in N^{1, p}(X)$, then $u \sim v$ if and only if $u=v$ q.e. Moreover, if $u, v \in N^{1, p}(X)$ and $u=v$ a.e., then $u=v$ q.e. Capacity is also important for the following two notions, which are central in this paper.

Definition 2.3. A set $U \subset X$ is quasiopen if for every $\varepsilon>0$ there is an open set $G \subset X$ such that $C_{p}(G)<\varepsilon$ and $G \cup U$ is open. A function $u$ defined on a set $E \subset X$ is quasicontinuous if for every $\varepsilon>0$ there is an open set $G \subset X$ such that $C_{p}(G)<\varepsilon$ and $\left.u\right|_{E \backslash G}$ is finite and continuous. 
The quasiopen sets do not in general form a topology, see Remark 9.1 in BjörnBjörn [2]. However it follows easily from the countable subadditivity of $C_{p}$ that countable unions and finite intersections of quasiopen sets are quasiopen. (We consider finite sets to be countable throughout the paper.) For characterizations of quasiopen sets and quasicontinuous functions see Björn-Björn-Malý [5].

Definition 2.4. We say that $X$ supports a $p$-Poincaré inequality if there exist constants $C>0$ and $\lambda \geq 1$ such that for all balls $B \subset X$, all integrable functions $f$ on $X$ and all $p$-weak upper gradients $g$ of $f$,

$$
f_{B}\left|f-f_{B}\right| d \mu \leq C \operatorname{diam}(B)\left(f_{\lambda B} g^{p} d \mu\right)^{1 / p},
$$

where $f_{B}:=f_{B} f d \mu:=\int_{B} f d \mu / \mu(B)$.

In $\mathbf{R}^{n}$ equipped with a doubling measure $d \mu=w d x$, where $d x$ denotes Lebesgue measure, the $p$-Poincaré inequality (2.2) is equivalent to the $p$-admissibility of the weight $w$ in the sense of Heinonen-Kilpeläinen-Martio [9], see Corollary 20.9 in [9] and Proposition A.17 in [1]. Moreover, in this case $g_{u}=|\nabla u|$ if $u \in N^{1, p}\left(\mathbf{R}^{n}\right)$.

\section{Fine topology}

Throughout the rest of the paper, we assume that $X$ is complete and supports a $p$-Poincaré inequality, and that $\mu$ is doubling. We also assume that $U$ is quasiopen.

In this section we recall the basic facts about the fine topology associated with Sobolev spaces and prove some auxiliary results which will be crucial in the subsequent sections. Recall that $\operatorname{cap}_{p}(E, B)=\inf _{u} \int_{X} g_{u}^{p} d \mu$, where $E \subset B$ and the infimum is taken over all $u \in N_{0}^{1, p}(B)$ such that $u \geq 1$ on $E$.

Definition 3.1. A set $E \subset X$ is thin at $x \in X$ if

$$
\int_{0}^{1}\left(\frac{\operatorname{cap}_{p}(E \cap B(x, r), B(x, 2 r))}{\operatorname{cap}_{p}(B(x, r), B(x, 2 r))}\right)^{1 /(p-1)} \frac{d r}{r}<\infty .
$$

A set $V \subset X$ is finely open if $X \backslash V$ is thin at each point $x \in V$.

In the definition of thinness, we make the convention that the integrand is 1 whenever $\operatorname{cap}_{p}(B(x, r), B(x, 2 r))=0$. It is easy to see that the finely open sets give rise to a topology, which is called the fine topology. Every open set is finely open, but the converse is not true in general. A function $u: V \rightarrow[-\infty, \infty]$, defined on a finely open set $V$, is finely continuous if it is continuous when $V$ is equipped with the fine topology and $[-\infty, \infty]$ with the usual topology. See $[1$, Section 11.6] and [3] for further discussion on thinness and the fine topology.

The following definition will play an important role in this paper.

Definition 3.2. A set $E \subset A$ is a p-strict subset of $A$ if there is a function $u \in N_{0}^{1, p}(A)$ such that $u=1$ on $E$.

Equivalently, it can in addition be required that $0 \leq u \leq 1$, as in KilpeläinenMalý [12]. The following lemma shows that there are many nice $p$-strict subsets of finely open sets. They play the role of relatively compact subsets of open sets. In particular, there is a base of fine neighbourhoods, consisting only of $p$-strict subsets. Recall that $W \Subset V$ if $\bar{W}$ is a compact subset of $V$.

Lemma 3.3. Let $V$ be finely open and let $x_{0} \in V$. Then there exist a finely open $W \ni x_{0}$ and an upper semicontinuous finely continuous function $v \in N_{0}^{1, p}(V)$ 
with compact support in $V$ such that $0 \leq v \leq 1$ everywhere and $v=1$ in $W$. In particular, $W$ is a $p$-strict subset of $V$ and $W \Subset V$.

Proof. Since $V$ is finely open, $E:=X \backslash V$ is thin at $x_{0}$. By the weak Cartan property in Björn-Björn-Latvala [3, Theorem 5.1] there exists a lower semicontinuous finely continuous function $u \in N^{1, p}(B)$ in a ball $B \ni x_{0}$ such that $0<u \leq 1$ in $B$, $u=1$ in $E \cap B$ and $u\left(x_{0}\right)<1$. Let $0 \leq \eta \leq 1$ be a Lipschitz function with compact support in $B$ such that $\eta\left(x_{0}\right)=1$. Then $f=\eta(1-u) \in N_{0}^{1, p}(V)$ is upper semicontinuous and finely continuous in $X$ and $f\left(x_{0}\right)=1-u\left(x_{0}\right)>0$.

To conclude the proof, set

$$
W=\left\{x \in V: f(x)>\frac{1}{2} f\left(x_{0}\right)\right\} \quad \text { and } \quad v(x)=\min \left\{1,\left(\frac{4 f(x)}{f\left(x_{0}\right)}-1\right)_{+}\right\} .
$$

A simple calculation shows that $v=1$ in $W$, and the upper semicontinuity of $f$ implies that $W \Subset \operatorname{supp} v \Subset V$. As $f$ is finely continuous, $W$ is finely open.

The following principle will play an important role in the later sections.

Theorem 3.4. (Quasi-Lindelöf principle) For each family $\mathcal{V}$ of finely open sets there is a countable subfamily $\mathcal{V}^{\prime}$ such that

$$
C_{p}\left(\bigcup_{V \in \mathcal{V}} V \backslash \bigcup_{V^{\prime} \in \mathcal{V}^{\prime}} V^{\prime}\right)=0
$$

The proof is very similar to the proof in Heinonen-Kilpeläinen-Malý [8, Theorem 2.3] for unweighted $\mathbf{R}^{n}$, invoking the fine Kellogg property for metric spaces from Björn-Björn-Latvala [4, Corollary 1.3]. We leave the details to the interested reader.

The proof of the fine Kellogg property in [4] depends in turn on the Choquet property, the Cartan property and ultimately on the Cheeger differentiable structure. It would be nice if one could obtain a proof of the quasi-Lindelöf principle which does not depend on the Cheeger structure.

\section{Local Newtonian spaces and quasicontinuity}

Solutions of differential equations and variational problems are usually considered in local Sobolev spaces. On a quasiopen set $U$ there are at least three different natural candidates for a local Newtonian space.

Definition 4.1. A family $\mathcal{B}$ of quasiopen sets is a quasicovering of $U$ if it is countable, $\bigcup_{V \in \mathcal{B}} V \subset U$ and $C_{p}\left(U \backslash \bigcup_{V \in \mathcal{B}} V\right)=0$. If every $V \in \mathcal{B}$ is a finely open $p$-strict subset of $U$ and $\bar{V} \Subset U$, then $\mathcal{B}$ is a $p$-strict quasicovering of $U$. Moreover, we say that

(i) $u \in N_{\text {fine-loc }}^{1, p}(U)$ if $u \in N^{1, p}(V)$ for all finely open $p$-strict subsets $V \Subset U$;

(ii) $u \in N_{\text {quasi-loc }}^{1, p}(U)$ (resp. $L_{\text {quasi-loc }}^{p}(U)$ ) if there is a quasicovering $\mathcal{B}$ of $U$ such that $u \in N^{1, p}(V)$ (resp. $\left.u \in L^{p}(V)\right)$ for all $V \in \mathcal{B}$.

Recall that $f \in N_{\text {loc }}^{1, p}(U)$ if for every $x \in U$ there exists a ball $B_{x} \ni x$ such that $f \in N^{1, p}\left(B_{x} \cap U\right)$. The space $N_{\text {loc }}^{1, p}(U)$ is natural in connection with Newtonian spaces, but on $\mathbf{R}^{n}$ it is not even obvious how to define classical (nonlocal) Sobolev spaces on quasiopen sets. A fruitful definition of $W^{1, p}(U)$ was given by Kilpeläinen-Malý [12] and they also introduced a local space (called $W_{\text {loc }}^{1, p}(U)$ therein) which is similar to our definition of $N_{\text {fine-loc }}^{1, p}(U)$. The same definitions were used in Latvala [14]. Yet 
another definition of a local space was considered by Malý-Ziemer [15, p. 149] (and called $W_{p \text {-loc }}^{1, p}(U)$ ). It inspired our definition of $N_{\text {quasi-loc }}^{1, p}(U)$. It would be interesting to know how $N_{\text {quasi-loc }}^{1, p}(U)$ is related to $W_{p \text {-loc }}^{1, p}(U)$.

Proposition 4.2. There exists a $p$-strict quasicovering $\mathcal{B}$ of $U$ consisting of finely open sets. Moreover, the Newtonian functions associated with the $p$-strict subsets in $\mathcal{B}$ can be chosen compactly supported in $U$.

Proof. By Theorem 4.9 (a) in Björn-Björn-Latvala [3] we can write $U=V \cup E$, where $V$ is finely open and $C_{p}(E)=0$. For every $x \in V$, Lemma 3.3 provides us with a finely open set $V_{x} \ni x$ such that $V_{x} \Subset V$ and the corresponding function $v_{x} \in N_{0}^{1, p}(V)$ has compact support in $V$. The collection $\mathcal{B}^{\prime}=\left\{V_{x}\right\}_{x \in V}$ covers $V$ and by the quasi-Lindelöf principle (Theorem 3.4), and the fact that $C_{p}(E)=0$, there exists a countable subcollection $\mathcal{B} \subset \mathcal{B}^{\prime}$ such that $C_{p}\left(U \backslash \bigcup_{V_{x} \in \mathcal{B}} V_{x}\right)=0$.

We will also need the following result, which is a special case of Corollary 3.7 in Björn-Björn [2] in view of Lemmas 9.2 and 9.3 in [2].

Lemma 4.3. Let $U$ be quasiopen and $u \in N_{\text {loc }}^{1, p}(X)$. Then $g_{u, U}=g_{u}$ a.e. in $U$, where $g_{u, U}$ and $g_{u}$ are the minimial $p$-weak upper gradients of $u$ taken with respect to $U$ and $X$ as ambient space, respectively.

Using Lemma 4.3 and Proposition 4.2 it is fairly easy to see that $N_{\text {loc }}^{1, p}(U) \subset$ $N_{\text {fine-loc }}^{1, p}(U) \subset N_{\text {quasi-loc }}^{1, p}(U)$. For open $U$, we have $N_{\text {loc }}^{1, p}(U)=N_{\text {fine-loc }}^{1, p}(U)$, but we do not know if this holds for quasiopen $U$. Note that in general $N_{\text {loc }}^{1, p}(U) \neq N_{\text {quasi-loc }}^{1, p}(U)$ even when $U$ is open. Consider e.g. the unit ball $B \subset \mathbf{R}^{n}$ with $n \geq p$, for which $N_{\text {loc }}^{1, p}(B) \varsubsetneqq N_{\text {loc }}^{1, p}(B \backslash\{0\}) \subset N_{\text {quasi-loc }}^{1, p}(B)$.

We are now ready to prove Theorem 1.2 , which we obtain in the following more general form.

Theorem 4.4. Assume that $u \in N_{\text {quasi-loc }}^{1, p}(U)$. Then $u$ is finite q.e. and finely continuous q.e. in $U$. In particular, $u$ is quasicontinuous in $U$.

Proof. By assumption there is a quasicovering $\mathcal{B}$ of $U$ such that $u \in N^{1, p}(\widetilde{U})$ for each $\widetilde{U} \in \mathcal{B}$. By Proposition 4.2 , for each $\widetilde{U} \in \mathcal{B}$ there exists a $p$-strict quasicovering $\mathcal{B}_{\widetilde{U}}$ of $\widetilde{U}$ consisting of finely open $p$-strict subsets $V_{j, \widetilde{U}}$ such that $\bar{V}_{j, \widetilde{U}} \Subset \widetilde{U}$.

First, assume that $u$ is bounded and let $V=V_{j, \widetilde{U}}$ be arbitrary. Then there is $v \in N_{0}^{1, p}(\widetilde{U})$ with $v=1$ on $V$ and $0 \leq v \leq 1$ everywhere. Let $w=v u$, extended by 0 outside of $\widetilde{U}$. Then $w \in N^{1, p}(\widetilde{U})$ by Lemma 2.6 in Björn-Björn-Latvala [4]. As $|w| \leq C v \in N_{0}^{1, p}(\widetilde{U})$ (with $C=\sup _{U}|u|<\infty$ ), it follows from [1, Lemma 2.37] that $w \in N_{0}^{1, p}(\widetilde{U}) \subset N^{1, p}(X)$, and in particular $w$ is quasicontinuous in $X$. By Theorem 4.9 (b) in Björn-Björn-Latvala [3], $w$ is finely continuous q.e. in $X$. Since $u=w$ in the finely open set $V$, it follows that $u$ is finely continuous q.e. in $V$, and thus q.e. in $U$, as $V$ was arbitrary.

If $u$ is arbitrary, let $u_{k}=\max \{\min \{u, k\},-k\}, k=1,2, \ldots$, be the truncations of $u$ at levels $\pm k$. By the first part of the proof there is a set $E_{k}$ such that $C_{p}\left(E_{k}\right)=0$ and $u_{k}$ is finely continuous at all $x \in U \backslash E_{k}$.

By Proposition 1.30 in [1], $u$ is finite q.e. in $\widetilde{U}$ for each $\widetilde{U} \in \mathcal{B}$. As $\mathcal{B}$ is a quasicovering, there is a set $E_{0}$ with $C_{p}\left(E_{0}\right)=0$ such that $u$ is finite in $U \backslash E_{0}$. Let $E=\bigcup_{j=0}^{\infty} E_{j}$, which is a set with zero capacity. If $x \in U \backslash E$, then $u(x)$ is finite and hence there is $k$ such that $|u(x)|<k$. Since $u_{k}$ is finely continuous at $x$ and $|u(x)|<k$, 
we conclude that $u$ is also finely continuous at $x$. Hence $u$ is finely continuous q.e. in $U$. The quasicontinuity of $u$ now follows from [4, Theorem 1.4(b)].

\section{Sobolev spaces based on fine upper gradients}

The main aim of this section is to prove Theorem 1.1. To do so it will be convenient to make the following definition, which has been inspired by the definition of fine gradients in $\mathbf{R}^{n}$ from Kilpeläinen-Malý [12]. Recall that $U$ is quasiopen and $g_{u, V}$ is the minimal $p$-weak upper gradient of $u$ taken with respect to $V$ as the ambient space.

Definition 5.1. A function $\tilde{g}_{u}: U \rightarrow[0, \infty]$ is a $p$-fine upper gradient of $u \in$ $N_{\text {quasi-loc }}^{1, p}(U)$ if there is a quasicovering $\mathcal{B}$ of $U$ such that $u \in N^{1, p}(V)$ for every $V \in \mathcal{B}$ and $\tilde{g}_{u}=g_{u, V}$ a.e. in $V$.

The following result shows that $p$-fine upper gradients always exist.

Lemma 5.2. If $u \in N_{\text {quasi-loc }}^{1, p}(U)$, then it has a unique $p$-fine upper gradient $\tilde{g}_{u}$.

The uniqueness is up to a.e. Moreover, by Definition 5.1, if $g: U \rightarrow[0, \infty]$ satisfies $g=\tilde{g}_{u}$ a.e., then $g$ is also a $p$-fine upper gradient of $u$.

Proof. Let $\mathcal{B}$ be a quasicovering of $U$ such that $u \in N^{1, p}(V)$ for every $V \in \mathcal{B}$. If $V, W \in \mathcal{B}$, then $V \cap W$ is quasiopen and Lemma 4.3 shows that

$$
g_{u, V}=g_{u, V \cap W}=g_{u, W} \quad \text { a.e. in } V \cap W .
$$

We can therefore define $\tilde{g}_{u}: U \rightarrow[0, \infty]$ so that $\tilde{g}_{u}=g_{u, V}$ a.e. in $V$ for every $V \in \mathcal{B}$. By definition, $\tilde{g}_{u}$ is a $p$-fine upper gradient of $u$.

To prove the uniqueness, assume that $g$ is any $p$-fine upper gradient of $u$, and let $\mathcal{B}$ and $\mathcal{B}^{\prime}$ be the quasicoverings given in Definition 5.1 for $\tilde{g}_{u}$ and $g$, respectively. Let $V \in \mathcal{B}$ and $W \in \mathcal{B}^{\prime}$. Since $V \cap W$ is quasiopen, Lemma 4.3 then yields that

$$
\tilde{g}_{u}=g_{u, V}=g_{u, V \cap W}=g_{u, W}=g \quad \text { a.e. in } V \cap W .
$$

As $\mathcal{B}^{\prime}$ and $\mathcal{B}$ are quasicoverings it follows that $\tilde{g}_{u}=g$ a.e. in $V$, and thus in $U$. This proves the a.e. uniqueness of $\tilde{g}_{u}$.

Next, we show that $p$-fine upper gradients are the same as minimal $p$-weak upper gradients, with minimality in the appropriate sense. Note that minimality has been built into the definition of $p$-fine upper gradients.

Theorem 5.3. Let $u \in N_{\text {quasi-loc }}^{1, p}(U)$ and let $\tilde{g}_{u}$ be a $p$-fine upper gradient of $u$. Then $\tilde{g}_{u} \in L_{\text {quasi-loc }}^{p}(U)$ and it is a $p$-weak upper gradient of $u$ in $U$ which is minimal in the following two senses:

(a) If $W \subset U$ is quasiopen and $u \in N_{\text {loc }}^{1, p}(W)$, then $\tilde{g}_{u}=g_{u, W}$ a.e. in $W$.

(b) If $g \in L_{\text {quasi-loc }}^{p}(U)$ is a $p$-weak upper gradient of $u$, then $\tilde{g}_{u} \leq g$ a.e.

Proof. Let $\mathcal{B}$ be the quasicovering of $U$ associated with $\tilde{g}_{u}$ as in Definition 5.1. It is immediate that $\tilde{g}_{u} \in L_{\text {quasi-loc }}^{p}(U)$. Next we show that $\tilde{g}_{u}$ is a $p$-weak upper gradient of $u$ in $U$. Since $C_{p}\left(U \backslash \bigcup_{V \in \mathcal{B}} V\right)=0$, we conclude from Proposition 1.48 in [1] and Remark 3.5 in Shanmugalingam [17] that $p$-almost every curve $\gamma$ in $U$ avoids $U \backslash \bigcup_{V \in \mathcal{B}} V$ and is such that $\gamma^{-1}(V)$ is open for each $V \in \mathcal{B}$. Moreover, by [1, Lemma 1.40], we may assume that $\tilde{g}_{u}$ satisfies (2.1) on any subcurve $\tilde{\gamma} \subset \gamma$, whose image is contained in $V$. Let $\gamma:\left[0, l_{\gamma}\right] \rightarrow \bigcup_{V \in \mathcal{B}} V$ be such a curve.

Since each $\gamma^{-1}(V)$ is open, it is a countable union of (relatively) open subintervals of $\left[0, l_{\gamma}\right]$, whose collection for all $V \in \mathcal{B}$ covers $\left[0, l_{\gamma}\right]$. By compactness, $\left[0, l_{\gamma}\right]$ can be 
covered by finitely many such intervals. We can then find slightly smaller closed intervals so that $\left[0, l_{\gamma}\right]=\bigcup_{j=1}^{N}\left[a_{j}, b_{j}\right]$, where $\left[a_{j}, b_{j}\right] \subset \gamma^{-1}\left(V_{j}\right)$ for some $V_{j} \in \mathcal{B}$. Since this is a finite union of intervals we can, by decreasing their lengths, assume that $0=a_{1}<b_{1}=a_{2}<\ldots<b_{N}=l_{\gamma}$. We then have

$$
\left|u(\gamma(0))-u\left(\gamma\left(l_{\gamma}\right)\right)\right| \leq \sum_{j=1}^{N}\left|u\left(\gamma\left(a_{j}\right)\right)-u\left(\gamma\left(b_{j}\right)\right)\right| \leq \sum_{j=1}^{N} \int_{\left.\gamma\right|_{\left[a_{j}, b_{j}\right]}} \tilde{g}_{u} d s=\int_{\gamma} \tilde{g}_{u} d s .
$$

This shows that $\tilde{g}_{u}$ is a $p$-weak upper gradient of $u$ in $U$. We now turn to the minimality.

(a) If $V \in \mathcal{B}$, then $V \cap W$ is quasiopen. Hence by Lemma 4.3,

$$
g_{u, W}=g_{u, V \cap W}=g_{u, V}=\tilde{g}_{u} \quad \text { a.e. in } V \cap W .
$$

As $\mathcal{B}$ is a quasicovering, we see that $g_{u, W}=\tilde{g}_{u}$ a.e. in $W$.

(b) Let $\widetilde{\mathcal{B}}$ be a quasicovering such that $g \in L^{p}(\widetilde{V})$ for each $\widetilde{V} \in \widetilde{\mathcal{B}}$. Then $u \in N^{1, p}(\widetilde{V})$ and by (a), $\tilde{g}_{u}=g_{u, \widetilde{V}} \leq g$ a.e. in $\widetilde{V}$. As $\widetilde{\mathcal{B}}$ is a quasicovering, it follows that $\tilde{g}_{u} \leq g$ a.e. in $U$.

Theorem 5.4. A function $u$ belongs to $N^{1, p}(U)$ if and only if $u \in L^{p}(U)$ and there is a $p$-fine upper gradient $\tilde{g}_{u} \in L^{p}(U)$. Moreover, in this case, $\tilde{g}_{u}=g_{u, U}$ a.e. in $U$.

Proof. If $u \in N^{1, p}(U)$, then $u \in L^{p}(U)$ and $g_{u, U} \in L^{p}(U)$. By Lemma 5.2, $u$ has a $p$-fine upper gradient $\tilde{g}_{u}$. Next, $\tilde{g}_{u}=g_{u, U}$ a.e., by Theorem 5.3, and hence $\tilde{g}_{u} \in L^{p}(U)$. Conversely, if $u \in L^{p}(U)$ and $\tilde{g}_{u} \in L^{p}(U)$ is a $p$-fine upper gradient $u$, then $\tilde{g}_{u}$ is a $p$-weak upper gradient of $u$ in $U$, by Theorem 5.3, and thus $u \in N^{1, p}(U)$.

The following result shows that in the definition of $p$-fine upper gradients, $u$ can be extended from $V$ to a global Newtonian function.

Proposition 5.5. If $u \in N_{\text {quasi-loc }}^{1, p}(U)$, then there is a $p$-strict quasicovering $\mathcal{B}$ of $U$ such that for every $V \in \mathcal{B}$ there exists $u_{V} \in N^{1, p}(X)$ with $u=u_{V}$ in $V$.

Proof. Let $\widetilde{\mathcal{B}}$ be a quasicovering of $U$ such that $u \in N^{1, p}(\widetilde{V})$ for every $\widetilde{V} \in \widetilde{\mathcal{B}}$. Theorem 4.4 shows that $u$ is quasicontinuous in $U$. Let $U_{k}=\{x \in U:|u(x)|<$ $k\}, k=1,2, \ldots$ By quasicontinuity, each $U_{k}$ is quasiopen and $\left\{U_{k}\right\}_{k=1}^{\infty}$ forms a quasicovering of $U$. For each $k$, let $\mathcal{B}_{k}=\left\{U_{k} \cap \widetilde{V}: \widetilde{V} \in \widetilde{\mathcal{B}}\right\}$ and for each $W \in \mathcal{B}_{k}$ use Proposition 4.2 to obtain a $p$-strict quasicovering $\mathcal{B}_{W}$ of $W$, together with the associated compactly supported functions in $W$. Then $\mathcal{B}=\bigcup_{k=1}^{\infty} \bigcup_{W \in \mathcal{B}_{k}} \mathcal{B}_{W}$ is a $p$-strict quasicovering of $U$, and $u$ is bounded on each $W \in \mathcal{B}$.

Next, let $k, W=U_{k} \cap \widetilde{V} \in \mathcal{B}_{k}$ and $V \in \mathcal{B}_{W}$ be arbitrary, and let $v \in N_{0}^{1, p}(W)$ be the associated function such that $v=1$ in $V$. Since $u \in N^{1, p}(W)$ is bounded in $W$ and $v$ has compact support in $W$, we see that $u \in N^{1, p}(\operatorname{supp} v)$. Lemma 2.6 in BjörnBjörn-Latvala [4] implies that $u v \in N^{1, p}(\operatorname{supp} v)$, and as $|u v| \leq k v \in N_{0}^{1, p}(\operatorname{supp} v)$, Lemma 2.37 in [1] then shows that $u v \in N_{0}^{1, p}(\operatorname{supp} v) \subset N^{1, p}(X)$. Let $u_{V}=u v \in$ $N^{1, p}(X)$ for $V \in \mathcal{B}$. Then $u=u_{V}$ in $V$.

The following definition is from Kilpeläinen-Malý [12], and has been our inspiration for Definition 5.1. Note however, that the metric space theory allows us to consider $u \in N^{1, p}(V)$ in Definition 5.1, which makes the situation simpler. In particular, we do not need to go outside of $U$ to define $p$-fine upper gradients. On the other hand, Proposition 5.5 shows that one can equivalently use functions $u_{V} \in N^{1, p}(X)$ 
such that $u=u_{V}$ in $V$. (In [12] a quasicovering may be uncountable, but it is required to contain a countable quasicovering in our sense. For the purpose of the definition below the existence of a quasicovering in either sense is obviously equivalent.)

Definition 5.6. Let $U \subset \mathbf{R}^{n}$. A function $u \in L^{p}(U)$ belongs to $W^{1, p}(U)$ if

(i) there is a quasicovering $\mathcal{B}$ of $U$ such that for every $V \in \mathcal{B}$ there is an open set $G_{V} \supset V$ and $u_{V} \in W^{1, p}\left(G_{V}\right)$ so that $u=u_{V}$ in $V$;

(ii) the fine gradient $\nabla u$, defined by $\nabla u=\nabla u_{V}$ a.e. on each $V \in \mathcal{B}$, also belongs to $L^{p}(U)$.

Moreover, we let

$$
\|u\|_{W^{1, p}(U)}=\left(\int_{U}\left(|u|^{p}+|\nabla u|^{p}\right) d x\right)^{1 / p} .
$$

As $\mathcal{B}$ is a quasicovering, the gradient $\nabla u$ is well defined a.e., and we may pick any representative. By Proposition 5.5 and Theorem 5.7 below, one can equivalently require that $G_{V}=\mathbf{R}^{n}$ in Definition 5.6.

Kilpeläinen-Malý [12] gave this definition for unweighted $\mathbf{R}^{n}$, but it makes sense also in weighted $\mathbf{R}^{n}$ (with a $p$-admissible weight), provided that we by $\nabla u_{V}$ mean the corresponding weighted Sobolev gradient, cf. the discussion on p. 13 in HeinonenKilpeläinen-Martio [9]. Theorem 5.7 below also holds, with the same proof, for weighted $\mathbf{R}^{n}$.

Theorem 5.7. Let $U \subset \mathbf{R}^{n}$. Then $u \in W^{1, p}(U)$ if and only if there exists $v \in N^{1, p}(U)$ such that $v=u$ a.e. in $U$. Moreover, $g_{v, U}=|\nabla u|$ a.e. in $U$ and $\|v\|_{N^{1, p}(U)}=\|u\|_{W^{1, p}(U)}$.

Proof. Assume that there is $v \in N^{1, p}(U)$ such that $v=u$ a.e. in $U$. By Proposition 5.5, there is a quasicovering $\mathcal{B}$ of $U$ such that for every $V \in \mathcal{B}$ there exists $v_{V} \in N^{1, p}\left(\mathbf{R}^{n}\right)$ with $v=v_{V}$ in $V$. By Proposition A.12 in [1], $v_{V} \in W^{1, p}\left(\mathbf{R}^{n}\right)$ and $g_{v_{V}}=\left|\nabla v_{V}\right|$ a.e. in $\mathbf{R}^{n}$. Hence $v \in W^{1, p}(U)$ and $\tilde{g}_{v}=|\nabla v|$ a.e. in $U$, where $\tilde{g}_{v}$ and $\nabla v$ are the $p$-fine and fine gradients of $v$, respectively. Theorem 5.4 implies that $g_{v, U}=\tilde{g}_{v}=|\nabla v|$ a.e. in $U$. As $u=v$ a.e., it follows directly that $u \in W^{1, p}(U)$ and $\nabla u=\nabla v$ a.e. Thus, $\|v\|_{N^{1, p}(U)}=\|v\|_{W^{1, p}(U)}=\|u\|_{W^{1, p}(U)}$.

Conversely, let $u \in W^{1, p}(U)$. Then there is a quasicovering $\mathcal{B}$ with $u_{V}$ and $G_{V}$ for $V \in \mathcal{B}$, as in Definition 5.6. By Theorem 4.4 in Heinonen-Kilpeläinen-Martio [9], for each $V \in \mathcal{B}$ there is a quasicontinuous function $\tilde{u}_{V}$ on the open set $G_{V}$ such that $\tilde{u}_{V}=u_{V}$ a.e. in $G_{V}$. By Proposition A.13 in [1], $\tilde{u}_{V} \in N^{1, p}\left(G_{V}\right)$.

If $V, W \in \mathcal{B}$, then $\tilde{u}_{V}=\tilde{u}_{W}$ q.e. in $G_{V} \cap G_{W}$, by Kilpeläinen [11] (or [1, Proposition 5.23]). Hence we can find functions $\tilde{u}: U \rightarrow[-\infty, \infty]$ and $g: U \rightarrow[0, \infty]$ such that

$$
\tilde{u}=\tilde{u}_{V} \text { q.e. in } V \text { and } g=g_{\tilde{u}_{V}, V} \text { a.e. in } V
$$

for every $V \in \mathcal{B}$. By definition, $g$ is a $p$-fine upper gradient of $\tilde{u}$. Lemma 4.3 above and Proposition A.12 in [1] yield that

$$
g=g_{\tilde{u}_{V}, V}=g_{\tilde{u}_{V}, G_{V}}=\left|\nabla \tilde{u}_{V}\right|=\left|\nabla u_{V}\right|=|\nabla u| \quad \text { a.e. in } V
$$

for every $V \in \mathcal{B}$, and hence $g=|\nabla u|$ a.e. in $U$. Since $|\nabla u| \in L^{p}(U)$, Theorem 5.4 implies that $\tilde{u} \in N^{1, p}(U)$. As $u=\tilde{u}$ a.e. in $U$, this concludes the proof.

If a function in $W^{1, p}(U)$ is changed on a set of measure zero, it remains in $W^{1, p}(U)$, in contrast to the Newtonian case. The following gives a more precise description of which representatives of $W^{1, p}(U)$ belong to $N^{1, p}(U)$. As it is valid in metric spaces 
we state it using the space

$$
\widehat{N}^{1, p}(U)=\left\{u: u=v \text { a.e. for some } v \in N^{1, p}(U)\right\} \text {. }
$$

Theorem 5.8. Let $u \in \widehat{N}^{1, p}(U)$. Then $u \in N^{1, p}(U)$ if and only if $u$ is quasicontinuous.

Proof. Assume that $u$ is quasicontinuous. As $u \in \widehat{N}^{1, p}(U)$ there is $v \in N^{1, p}(U)$ such that $v=u$ a.e. in $U$. By Theorem 4.4, $v$ is quasicontinuous. Hence by Kilpeläinen [11] (or [1, Proposition 5.23]) $u=v$ q.e., and thus also $u \in N^{1, p}(U)$. Here we also need to appeal to Proposition 5.22 in [1], which shows that our capacity satisfies Kilpeläinen's assumptions in [11].

The converse follows directly from Theorem 4.4.

Proof of Theorem 1.1. This follows directly from Theorems 5.7 and 5.8.

\section{References}

[1] BJÖRN, A., and J. BJÖRN: Nonlinear potential theory on metric spaces. - EMS Tracts Math. 17, Eur. Math. Soc., Zürich, 2011.

[2] BJÖRN, A., and J. BJöRN: Obstacle and Dirichlet problems on arbitrary nonopen sets in metric spaces, and fine topology. - Rev. Mat. Iberoam. 31, 2015, 161-214.

[3] BJÖRn, A., J. BJÖRN, and V. LATVALA: The weak Cartan property for the $p$-fine topology on metric spaces. - Indiana Univ. Math. J. 64, 2015, 915-941.

[4] BJörn, A., J. BJöRn, and V. Latvala: The Cartan, Choquet and Kellogg properties for the $p$-fine topology on metric spaces. - J. Anal. Math. (to appear).

[5] BJÖRN, A., J. BJÖRN, and J. MALÝ: Quasiopen and p-path open sets, and characterizations of quasicontinuity. - Preprint, 2015, arXiv:1509.02326.

[6] BJörn, A., J. BJöRn, and N. Shanmugalingam: Quasicontinuity of Newton-Sobolev functions and density of Lipschitz functions on metric spaces. - Houston J. Math. 34, 2008, 11971211.

[7] BJöRn, J.: Fine continuity on metric spaces. - Manuscripta Math. 125, 2008, 369-381.

[8] Heinonen, J., T. Kilpeläinen, and J. Malý: Connectedness in fine topologies. - Ann. Acad. Sci. Fenn. Ser. A I Math. 15, 1990, 107-123.

[9] Heinonen, J., T. Kilpeläinen, and O. Martio: - Nonlinear potential theory of degenerate elliptic equations. 2nd edition. - Dover, Mineola, NY, 2006.

[10] Heinonen, J., P. Koskela, N. Shanmugalingam, and J. T. Tyson: Sobolev spaces on metric measure spaces. - New Math. Monogr. 27, Cambridge Univ. Press, Cambridge, 2015.

[11] Kilpeläinen, T.: A remark on the uniqueness of quasi continuous functions. - Ann. Acad. Sci. Fenn. Math. 23, 1998, 261-262.

[12] Kilpeläinen, T., and J. Malý: Supersolutions to degenerate elliptic equation on quasi open sets. - Comm. Partial Differential Equations 17, 1992, 371-405.

[13] Korte, R.: A Caccioppoli estimate and fine continuity for superminimizers on metric spaces. - Ann. Acad. Sci. Fenn. Math. 33, 2008, 597-604.

[14] Latvala, V.: Finely superharmonic functions of degenerate elliptic equations. - Ann. Acad. Sci. Fenn. Math. Diss. 96, 1994.

[15] MalÝ, J., and W. P. Ziemer: Fine regularity of solutions of elliptic partial differential equations. - Amer. Math. Soc., Providence, RI, 1997.

[16] Shanmugalingam, N.: Newtonian spaces: An extension of Sobolev spaces to metric measure spaces. - Rev. Mat. Iberoam. 16, 2000, 243-279.

[17] Shanmugalingam, N.: Harmonic functions on metric spaces. - Illinois J. Math. 45, 2001, $1021-1050$.

Received 10 June $2015 \bullet$ Revised received 7 October 2015 • Accepted 19 October 2015 